\title{
Allocation of Tie-Line Costs in Power Exchange Scheduling Using a Multi-Agent Approach
}

\author{
Ping Wei $^{1} \quad$ Yonghe $\mathrm{Yan}^{2} \quad \mathrm{Yixin}^{\mathrm{Ni}^{1}} \quad$ Jerome Yen $^{3} \quad$ Felix F. Wu \\ ${ }^{1}$ Dept. of Electrical and Electronic Eng. \\ The University of Hong Kong \\ ${ }^{2}$ School of Comp. Sc., Telecom \& Inform. Syst. \\ Depaul University \\ Chicago, IL 60604
${ }^{3}$ Dept. of Syst. Eng. and Eng. Management Chinese University of Hong Kong \\ Hong Kong SAR, China
}

\begin{abstract}
After deregulations in power industries, the original boundary lines have been removed and how to support optimal cross-border electricity trade planning has become an important issue. Decentralization, or all market participants have the rights to participate in decision making, is one of the directions. In this paper a decentralized structure is suggested to solve the problem using multi-agent technology. In this structure the information centralization is prevented and each market participant behaves rationally based on local information. Although the market participants make decisions to protect its own benefits, the minimum production and transmission cost of the whole system can be reached finally. This structure is based on the method proposed in [5] and implemented on computer via a multi-agent system using Java programming language. A demonstration on a 5-area test system shows that the suggested new approach is effective and promising.
\end{abstract}

Keywords: power market, cross-border trade plan, decentralized optimization, multi-agent technology

\section{INTRODUCTION}

In recent years, power industry has been undergoing deregulations to introduce competitions among market participants in order to provide consumers with better quality of service (QoS) and more reasonable prices. The once centralized planning and decision making must now be adapted to the new market structure. Internet and related technologies, such as, World-wide Web (WWW), also supported the shaping of the new market structure, where more and more information or decisions about market are transmitted through Internet. Which increase the transparency and efficiency of market operation. Decentralization, or market participants have the rights to participate in the decision making, is one of the directions for utility companies to move.
Usually an interconnected power system consists of several regional networks with the tie-lines connected to each other. Under transmission open access, the electricity trade inside a regional system can be handled locally. While the electricity trades among regional systems should pay for the usage of tie lines and the regional networks along the transaction paths. It is clear that wholesale cross-border trades should be considered together with the transmission cost in advance with tie line capacity limits included. In this paper, we are using the European network as an example to study the wholesale cross-border trade planning, but the considerations are equally applicable for all the interconnected networks.

Electricity production in the European Union (EU) has for decades been based on monopoly production and 15 separate, national markets. Community Directive 96/92/EC has brought about a change to develop the common electricity market in Europe [1]. However there are several considerations for the common rule of transmission open access: (1) In order to schedule such wholesale crossborder trade, a central transmission system operator (TSO) seems to be necessary to collect all the information and do the calculations. Although for system security purposes it seems to be necessary anyway, the member TSOs may be opposed to this idea mostly for political reasons. (2) It is hard to ask for extreme equality when the national transmission systems facing native demands and foreign demands at the same time. How to obtain the optimum with the consideration of priority?

Generally there are two basic approaches to handle the wholesale cross-border trade schedule. One is the central schedule approach where the market operator is responsible to work out an optimal cross-border transmission schedule at minimum transmission cost with consideration of system operation constraints and then allocates the cost to individual transactions. Centralized optimization methods can be used for the first task, such as Ref. [2] and [3]. Very often market participants might doubt the fairness of the centrally announced results. Besides the central optimization has to be re-calculated whenever a new transaction is added. The other approach is the decentralized schedule, which uses the invisible market hand to solve the problem by market participants themselves. One such implementation [4] uses the Bilateral Shapley Value to negotiate in multilateral trades via a 
multi-agent system. This approach avoids the centralized decision making of the market operator and is quite attractive to market participants. However the optimal social welfare is not guarantied. In Ref. [5], a decentralized method is suggested base on 'first come, first serve' rule to implement the cross-border trade planning with the help of multi-agent technology. However in order to keep the system security and improve the speed, a central sever is still required.

In this paper, we are going to suggest a decentralized structure based on the method proposed in [5]. In the new structure, the centralized information collection is prevented and each regional system acts rationally based on local information to search its own benefits. However the total transmission cost is minimized after such selfprotective decisions. The significant advantage of this structure lies in that it is based on each participant's rational behavior and any super controller can be avoided.

The paper is organized as follows: in next section, we shall first present the mathematical model of the problem in its centralized optimal format. Then our decentralized approach is introduced. In section III, we give out detailed model of multi-agent system. System implementations and complexities are introduced in Section IV. In section V, we use a 5-area example system to illustrate negotiations among participants. Conclusions are made in the last section.

\section{MATHEMATICAL MODEL}

The basic assumptions used in our study are as follows:

(a) The transmission price of each tie line is a constant and announced in Sper unit power flow. The transmission limits of tie lines are known in per unit. For simplicity, the transmission loss is neglected (Its cost can be included approximately into the tie line transmission price) and the power flow of each tie line is controllable.

(b) An area is clarified as supply, demand or transit area if its net injection power $P_{i}$ is greater than, less than or equal to zero. $P_{i, \max }$ is the net injection capacity of area $i$ when $P_{i}>0$. The net generation capacity $P_{i, \max }$ for a supply area and the load demand of a demand area are all known.

(c) There are enough generation capacities to meet the load demands in the entire inter-connected system and the tie-line capacity is enough such that all the demands can be satisfied via proper schedule.

For the wholesale cross-border trades scheduling problem, the math model for the centralized optimal decision can be formulated as follows:

$$
\begin{aligned}
& \min \sum_{(i, j) \in \mathrm{A}} c_{i j} \cdot f_{i j}+\sum_{i \in S}\left(t_{i} \cdot \sum_{j:(i, j) \in A} f_{i j}\right)+\sum_{i \in D, T}\left(t_{i} \cdot \sum_{k:(k, i) \in A} f_{k i}\right) \\
& \text { s.t. } \sum_{j:(i, j) \in A} f_{i j}-\sum_{k:(k, i) \in A} f_{k i}=P_{i} \text { for } i=1,2, \ldots, n \\
& 0 \leq f_{\mathrm{ij}} \leq f_{i j, \max } \\
& \text { for all }(i, j) \in A \\
& 0 \leq P_{i} \leq P_{i, \max } \\
& \text { for all } i \in S
\end{aligned}
$$

where

$f_{i j}$ : $\quad$ power flow on the tie-line from region $i$ to region $j$, and $f_{i j}>0$;

$f_{i j, \max }$ : capacity of the lie-line from region $i$ to region $j$;

$c_{i j}: \quad$ price of per unit power flow for usage of tie-line $(i, j)$;

$t_{i}$ : price of per unit power flow for usage of network of area $i$

$S, D, T$ : denote supply, demand and transit area sets respectively;

$A$ : $\quad$ entire tie line set with $\mathrm{m}$ directed tie-line flows;

$n: \quad$ total number of areas.

The three terms of the objective function in (1) are the total costs for tie-lines usage; the total fees for usage of all supply area networks; and the total fees for the usage of other area networks respectively. It should be noticed that the generation cost is not included in the objective function for simplicity. There is no difficulty to include generation cost of each supply area into the problem. This is realized through introducing a fictitious 'supply area - tie line' set with the tie line transmission price equal to the generation cost of the supply area and the original supply area becomes a 'transit' area in the new system.

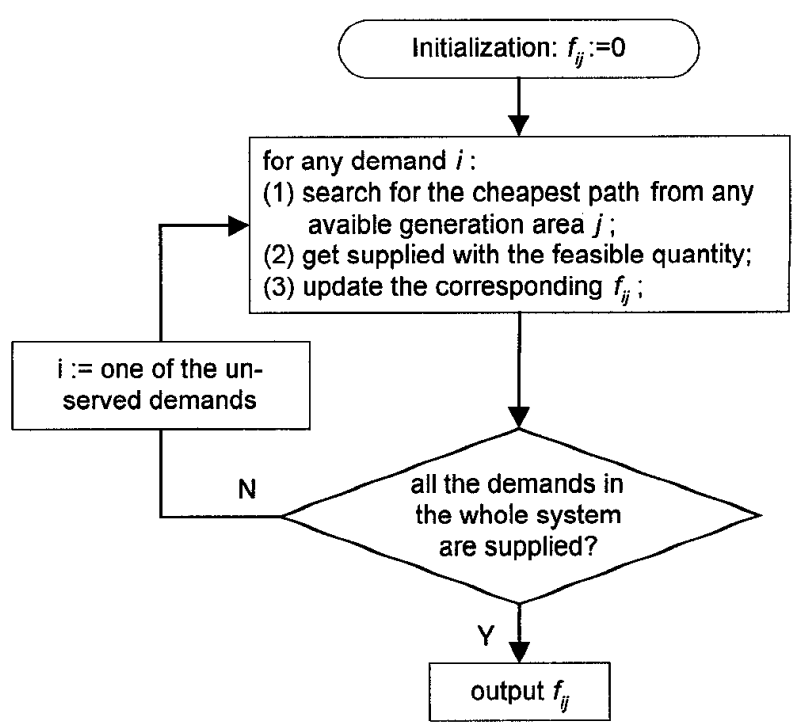

Figure1: flow chart of the decentralized approach

A decentralized approach is proposed in Ref. [5] to solve the linear optimization problem (1). The basic idea is that within each round of iteration, one demand $i$ searches for the cheapest path to get supplied from any available 
generation area $j$. The quantity of the supply is the minimal value of following three parameters: the un-served demand of area $i$, the available generating capacity of area $j$, and the available transmission capacity of path from area $j$ to area $i$. This process is repeated again and again all the loads in the whole system are totally satisfied. The flow chart is shown in figure 1. Using the inductive method, we proved that the final flow of the network from the decentralized approach above is the same as that from the centralized optimization defined in (1). Detailed algorithm and proof can be found in [5].

The advantages of the new approach are apparent:

(a) There is no need for a central coordinator. Each demand area searches for the cheapest path to satisfy its own need.

(b) Every demand area is satisfied with its choice based on the available cheapest path and doesn't need to worry about the bias from central processing.

(c) The minimal total transmission cost can still be guaranteed at the end with system constraints satisfied.

(d) When a new trade is added, previous trade schedules will not change. This is extremely attractive as compared with centralized optimization approaches.

(e) The new approach does not need transmission cost allocation calculation since it can obtain transmission cost of each trade during the process.

(f) The area power generation cost can also be included easily by introducing a fictitious 'supply area - tie line' set as mentioned before.

\section{MULTI-AGENT SYSTEM MODELING}

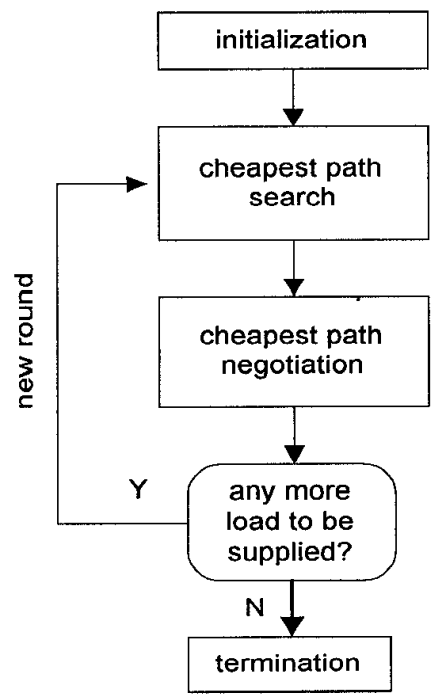

Figure 2: Functional Framework of the Multi-agent System

In our model each regional network is assumed to be a rational agent, who only has partial information, including the identity numbers of itself and the immediate neighbors, and the data of the tie-lines incident to itself, such as prices, capacities, and the existing flows. Each agent is responsible for updating related local information. We also assume that the communicate channels are perfect.

There are two major functions in the system by the communication of the agents. One is the cheapest path searching, the other is the cheapest path negotiation. The functional framework of this multi-agent system is shown in figure 2 .

Initialization:

Each agent collects the local information and sends the synchronizing message.

\section{Cheapest path search:}

A generic label-correcting algorithm [6] is modified to compute the cheapest path by successively updating the cost labels. Each agent maintains a set of cost labels $p($.) at every stage. The label $p(j)$ is either $\propto$, indicating that it has yet to discover a directed path from the source to agent $j$, or it is the cost of some directed path from the source to agent $j$. For each agent $j$ a predecessor index, pred( $j)$, is also maintained which records the agent prior to agent $j$ in the current directed path of cost $p(j)$. At termination, predecessor indices allow each agent to trace the cheapest path from the source node back to agent $j$. Detailed algorithm can be found in Ref. [6].

\section{Cheapest path negotiation:}

After each agent knows the identity number of its preceding agent along the cheapest path, it will send the message to the preceding agent for the usage of that regional network. There are three actions for a rational agent when receiving such request: (1) selfish plan when the receiver is a deficit agent, i.e., to decline the requests and refuse other agents to use its own regional networks until its own loads are fully supplied; (2) modest plan when the receiver is a balanced agent, i.e., to pass on the received requests to the preceding agent on the cheapest path and allow other agents to use its own regional networks by charging transit fees. (3) ego-centric plan when the receiver is a excess agent, i.e., to accept the requests based on the rule of "first come and first serve" and allow other agents to use its own networks.

Termination:

When no agent sends request to other agents, that means all the loads are supplied, then the system terminates.

The advantages of this model lie in that:

(a) There is no centralized owner or controller of the global information about the network. Therefore, any agent does not depend on the centralized information to make decisions. 
(b) Each agent only has local information. Therefore it knows neither the global structure of the network, nor the numbers of agents in the network. This structure is quite attractive to develop a competitive market.

(c) The minimum transmission cost could be achieved by communication and cooperation among all agents.

(d) Each agent is satisfied with its choice since all the trades are resulted from rational behavior.

\section{IV.SYSTEM IMPLEMENTATION}

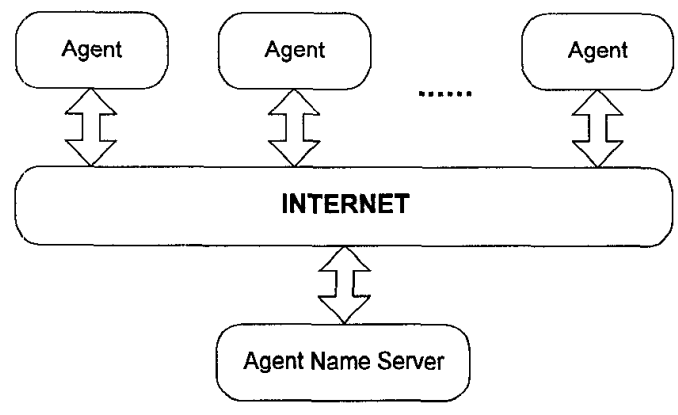

Figure 3 infrastructure of MASCAN

We have implemented a multi-agent system on the Internet - MASCAN (Multi-agent System for Cost Allocation on Network). Fig. 3 shows the infrastructure of MASCAN. Agent communication is done via the Internet. The agent name server provides agent registration service and the Internet connections for all agents. Fig. 4 shows a java applet and represents the agent $E$ of the test system in Section V. The agent name and password are used to register the agent into the agent name server.

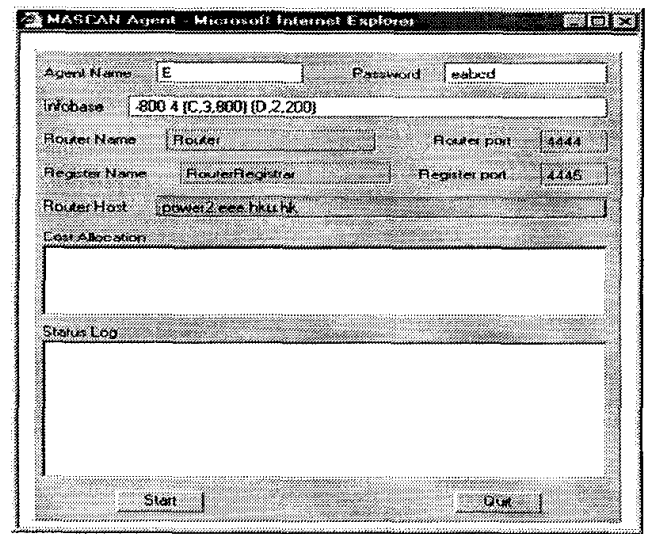

Figure 4 Applet of agent E

The execution time to process a message is always $O(I)$ in MASCAN. The worst case of execution in MASCAN is that each agent processes a message sequentially. Therefore, the time complexity of MASCAN equals to that of the message complexity. Hence we only need to analyze the message complexity.
The cheapest path computation needs to be executed one time within a round of negotiations. The message complexity of the cheapest path computation is $O\left(m^{2} n\right)$. There are at most $O\left(n D_{\max }\right)$ rounds of negotiation in MASCAN, where $D_{\max }$ is the largest demand of a given network. Finally, we can conclude that the message complexity and time complexity of MASCAN is $O\left(m^{2} n^{2} D_{\max }\right)$. (The time complexity will be much better in practice because all the agents may execute concurrently). Therefore the performance of the system is satisfactory.

\section{COMPUTER RESULTS}

A 5-area test system [7] (see Fig. 5) is used for computer test and to show how our method works based on multi-agent technology.

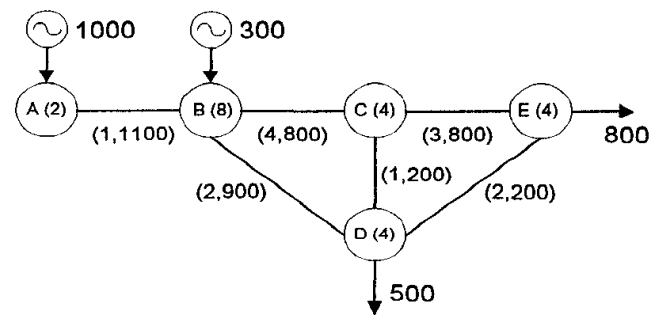

Figure 5 the test system schematic diagram

In Fig. 5 each bigger circle represents a regional network connected by tie lines to other networks. The number inside a circle represents the assumed transmission cost of per unit flow for transit through the regional systems. Each tie line has two parameters put in a parenthesis. The first number represents the transmission cost for per unit flow and the second number the transmission capacity of the tie line. The number by the side of a generator (or a demand) means the available generation capability (or the amount of load demand).

Region A and B are net exporters, they will execute the egocentric plan. Region $\mathrm{D}$ and $\mathrm{E}$ are net importers, so we assume they will execute the selfish plan when they are deficit, and then execute the modest plan when they become balanced. Region $\mathrm{C}$ is a transmit region with no net import/export, so it executes the modest plan.

\section{$1^{\text {st }}$ round}

The cheapest path from an excess agent (i.e., agent B) to agent $D$ is the path $B-D$ and the cheapest path from agent $B$ to agent $E$ is the path $B-D-E$. $E$ sends a request to $D$ to augment a flow of 800 units, and $D$ sends a request to $B$ to augment a flow of 500 units. Because agent $D$ is deficit area executing a selfish plan, it will reject the request from agent $\mathrm{E}$ to augment a flow via agent $\mathrm{D}$. While agent $\mathrm{B}$ is an excess agent who executes the egocentric plan. So B will accept the request by augmenting available 300 units 
through networks of $\mathrm{B}$ to agent $\mathrm{D}$, as illustrated by figure 6(a).

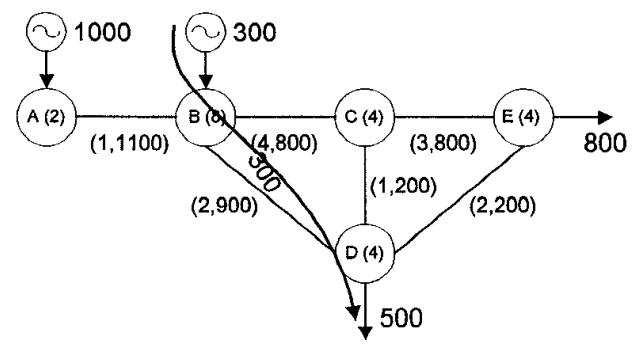

(a) Cost of trade B-D: $(8+2+4) \times 300=4200$

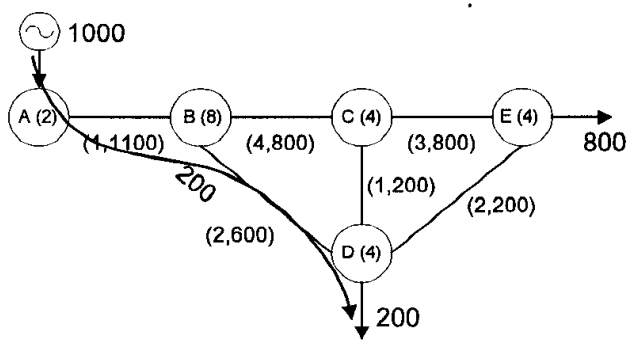

(b) Cost of trade A-B-D: $(2+1+8+2+4) \times 200=3400$

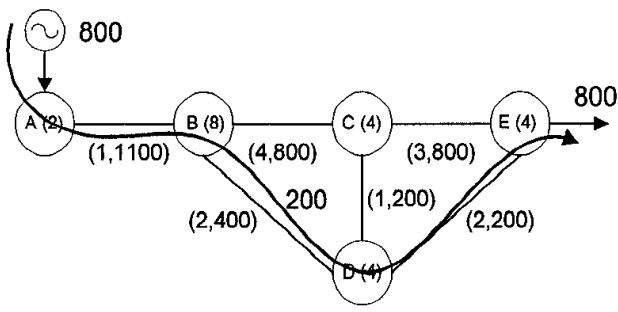

(c) Cost of trade A-B-D-E: $(2+1+8+2+4+2+4) \times 200=4600$

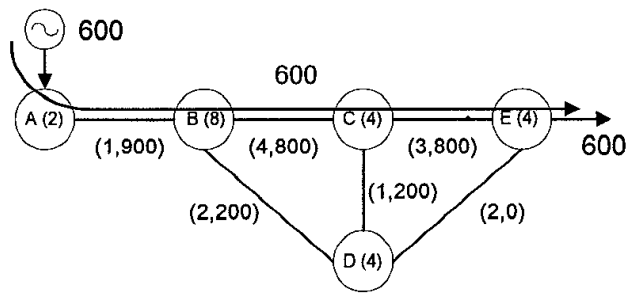

(d) Cost of trade A-B-C-E: $(2+1+8+4+4+3+4) \times 600=15600$

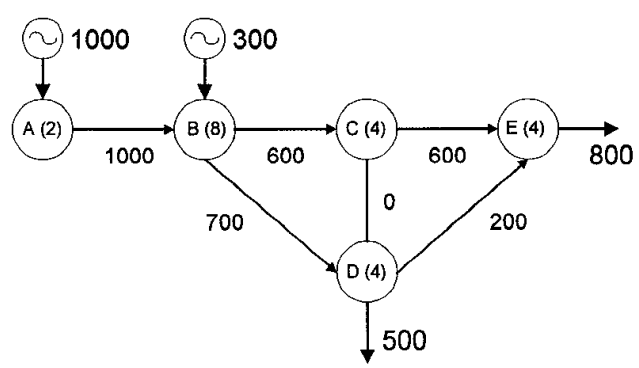

(e) Final solution of flows

Figure 6 Illustration of negotiation process $2^{\text {nd }}$ round

After the $1^{\text {st }}$ round, the cheapest path from an excess agent (i.e., agent $A$ ) to agent $D$ is the path A-B-D and the cheapest path from agent $A$ to agent $E$ is the path A-B-D-E. Because agent $D$ still is a deficit agent who is executing the selfish plan, it rejects the request from agent $E$ to augment a flow via agent $D$. While agent $B$ is a balanced agent who is executing the modest plan. It then passes agent D's request to A. Owing to the egocentric plan, agent A then approves the request to augment a flow of 200 units from agent $A$ to agent $\mathrm{D}$, as illustrated by figure $6(\mathrm{~b})$.

$3^{\text {rd }}$ round

After the $2^{\text {nd }}$ round, the cheapest path from the excess agent $A$ to agent $E$ is the path A-B-D-E. Since agent D becomes balanced, now all the agents (except $A$ ) along the path are the balanced agents who will execute modest plans. So the request sent by $E$ is passed on until it reaches $A$. Furthermore, all the regional networks along the path could be used at a transit fee. Because of the congestion of tieline D-E, only 200 units are augmented from agent $A$ to agent $\mathrm{E}$, as illustrated by figure $6(\mathrm{c})$.

$4^{\text {th }}$ round

After the $3^{\text {rd }}$ round, since the tie-line D-E has been fully used, the cheapest path from the excess agent $A$ to agent $E$ now is the path A-B-C-E. The balanced agents along the path (except A) merely pass on the requests received to their predecessors until agent $A$ gets the request. As the excess agent executing egocentric plan, $A$ then approves the request to augment 600 units from agent $A$ to agent $D$, as illustrated by figure $6(d)$.

Table 1: Cost Allocation (in money units)

\begin{tabular}{|c|c|c|c|}
\hline \multicolumn{2}{|c|}{ Cost flow } & Payment for service & Income from service \\
\hline \multirow{4}{*}{$\begin{array}{c}\text { Area } \\
\text { Network }\end{array}$} & $\mathrm{A}$ & 0 & 2000 \\
\cline { 2 - 4 } & $\mathrm{B}$ & 0 & 10400 \\
\cline { 2 - 4 } & $\mathrm{C}$ & 0 & 2400 \\
\cline { 2 - 4 } & $\mathrm{D}$ & 7600 & 2800 \\
\cline { 2 - 4 } & $\mathrm{E}$ & 20200 & 3200 \\
\hline \multirow{5}{*}{ Tie line } & A-B & 0 & 1000 \\
\cline { 2 - 4 } & $\mathrm{B}-\mathrm{C}$ & 0 & 2400 \\
\cline { 2 - 4 } & $\mathrm{C}-\mathrm{E}$ & 0 & 1800 \\
\cline { 2 - 4 } & B-D & 0 & 1400 \\
\cline { 2 - 4 } & $\mathrm{C}-\mathrm{D}$ & 0 & 0 \\
\cline { 2 - 4 } & E-D & 0 & 400 \\
\hline \multicolumn{2}{|c|}{ Total } & 27800 & 27800 \\
\hline
\end{tabular}

When negotiations end, the wholesale cross-border trades are finalized, and so is the cost of the trades. The final flow of trades for the whole system is shown in Fig. 6(e), and it is easy to check that the optimal cost of 27800 for the problem (1) is also achieved. A summary of the trade costs is listed in Table 1 . Totally agent $D$ needs to pay $3400+4200=7600$ by receiving 300 -units power from agent $\mathrm{B}$ and 200-units power from agent $\mathrm{A}$, while agent $\mathrm{E}$ needs 
to pay $4600+15600=20200$ by receiving 800 -units power from agent A going through two different paths. It is easy to prove the cost allocation result is the equilibrium point of the non-cooperative game for these five players [8].

\section{CONCLUSIONS}

In this paper, we have proposed a decentralized structure to make cross-border trade planning using multiagent technology under transmission open access. In the system, each agent represents a regional network, which acts rationally to protect its own benefit. Although each agent does not receive any centralized information to guarantee the autonomous behavior, the minimum cost of the whole system is achieved finally. This structure provides a theoretical basis for the electricity to join the Ecommerce. We have implemented a multi-agent system MASCAN by Java programming language. The demonstration on a 5 -area simple system shows that the approach is effective and promising.

\section{ACKNOWLEDGEMENT}

This research is supported by National Key Basic Research Special Fund (No. 1998020308 and 1998020305), RGC grant, HK SAR and CRCG grant, HKU, to whom sincere acknowledgements are expressed.

\section{REFERENCES}

[1] Janusz W. Bialek, "Tracing-Based Unifying Framework for Transmission Pricing of Cross-Border Trades in Europe", Proceedings of DRPT'2000, London, April 2000

[2] D. Shirmohammadi, P. R. Gribik, Eric T.K. Law, et al., "Evaluation of transmission network capacity use for wheeling transactions", IEEE Trans. On power Systems, Vol. 4, No. 4, October 1989

[3] Georage Gross, Shu Tao, "A Physical-Flow-Based Approach to Allocating Transmission Losses in a Transaction Framework", to be appeared in IEEE Trans. On Power Systems.
[4] Chris S.K. Yeung, Ada S.Y. Poon, Felix F. Wu, "Game Theoretical Multi-Agent Modelling of Coalition Formation for Multilateral Trades", IEEE Trans. On Power Systems, Vol. 14, No. 3, August 1999

[5] P. Wei, Y.H. Yan, J. Yen, Y.X. Ni, F. F. Wu, "A Decentralized Approach to the Optimal Wholesale Cross-border Trade Planning Using Multi-agent Technology", submitted to IEEE Trans. On Power Systems.

[6] Ahuja, R.K., Magnanti T.L., and J.B. Orlin, "Network Flows: Theory, Algorithms, and Applications", Printice Hall, Englewood Cliffs, New Jersey, 1993

[7] "International Exchanges of Electricity - Rules proposed by the European Transmission System Operators", 14.1.1999

[8] Yan Y.H., "A multi-agent based approach to transmission cost allocation", Doctoral Thesis, The University of Hong Kong, May 2000

\section{BIOGRAPHIES}

Ping Wei (Student M. IEEE) received her M. Eng. degree in electrical engineering from Southeast University, China. She is now a $\mathrm{Ph}$. D. student, Dept. of EEE, the University of Hong Kong. Her research interests include power system operation and power market.

Yonghe Yan received his $\mathrm{Ph}$. D. degree in computer science from the University of Hong Kong in 2000 . He now joins Depaul University as assistant professor. His research interests are E-commerce, AI and intelligent agents, distributed and collaborative computing.

Yixin Ni (S. M. IEEE) She received her B. Eng., M. Eng., and Dr. Eng. degrees all in electrical engineering from Tsinghua University, China. She was former professor and director of National Power System Lab, Tsinghua University and now with the University of Hong Kong. Her research interests are power system stability and control, FACTS, AI tech. applications in power systems and power market.

Jorome Yen received his $\mathrm{Ph}$. D. degree in systems engineering and management information systems from the University of Arizona in 1992. He now is an associate professor in Chinese University of Hong Kong. His major research interests include next generation Internet, E-commerce, digital library and information markets

Felix F. Wu (Fellow, IEEE) He received his Ph. D. degree from University of California at Berkeley (UCB). He is now a chair professor of electrical engineering and vice president of the University of Hong Kong. Prior to that he was a professor and vice-chair of Dept. of EECS, UCB. His research interests are electric energy industry restructuring, power system investment planning, design of modern control centres, distribution automation, distributed processing etc. 